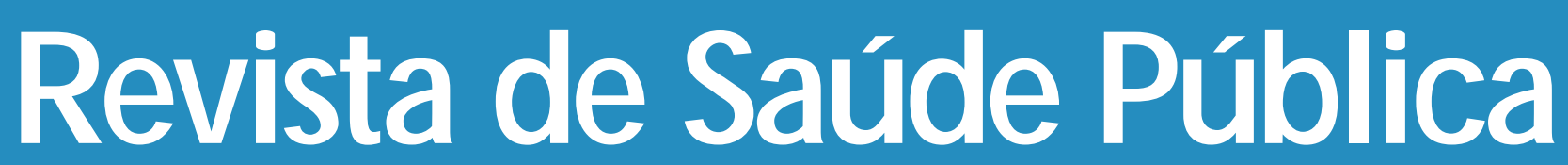

$\begin{array}{lllllll}J & 0 & \mathbf{U} & \mathbf{R} & \mathbf{N} & \mathbf{A} & \mathbf{L}\end{array}$

0 F

$\mathbf{P} \mathbf{U}$

B L I C

H E A L T H

\title{
Avaliação de programa educativo para portadores de glaucoma*
}

\section{Evaluation of an educational program for patients with glaucoma}

\section{Fernanda A. Cintra, Vital P. Costa, Jane A. G. Tonussi e N ewton Kara José}

Faculdade de Ciências M édicas da Universidade Estadual de Campinas (UNICAMP). Campinas, SP - Brasil (F.A.C., N.K.J.); Hospital das Clínicas da Faculdade de Medicina da Universidade de São Paulo. São Paulo, SP - Brasil (V.P.C.); Hospital das Clínicas da UNICAM P. Campinas, SP - Brasil (J.A.G.T.) 


\title{
Avaliação de programa educativo para portadores de glaucoma*
}

\section{Evaluation of an educational program for patients with glaucoma}

\author{
Fernanda A. Cintra, Vital P. Costa, Jane A. G. Tonussi e N ewton Kara José \\ Faculdade de Ciências Médicas da Universidade Estadual de Campinas (UNICAMP). Campinas, SP - \\ Brasil (F.A.C., N.K.J.); Hospital das Clínicas da Faculdade de Medicina da Universidade de São \\ Paulo. São Paulo, SP - Brasil (V.P.C.); Hospital das Clínicas da UNICAM P. Campinas, SP - Brasil \\ (J.A.G.T.)
}

\begin{abstract}
Resumo
Objetivo

Avaliar o resultado da implementação de um programa de educação à saúde a glaucomatosos; fornecer subsídios para a implantação de um programa de educação à saúde aos portadores de glaucoma atendidos em um serviço universitário.

Métodos Foi realizada entrevista individual com 50 portadores de glaucoma, através de questionário, seguida de reunião na qual se aplicou o programa de educação à saúde. Após um intervalo médio de 2,7 meses, realizou-se nova entrevista onde os portadores de glaucoma foram submetidos ao mesmo questionário, com o acréscimo de questões sobre a importância atribuída às orientações recebidas e sobre as modificações no autocuidado.

Resultados Não houve melhora significativa no conhecimento sobre a identificação da doença ocular, os efeitos colaterais, o significado do glaucoma, a importância do tratamento, a finalidade da terapêutica e o valor normal da pressão intra-ocular. Houve melhora significativa na técnica de instilação dos colírios e em relação ao conhecimento sobre a importância da hereditariedade e a finalidade da campimetria.

Conclusão Os autores concluem que o programa foi insuficiente para a apreensão de todo o seu conteúdo cognitivo. Os resultados podem ser conseqüentes ao tipo de processo ensino-aprendizagem, desenvolvido de forma vertical, que não atende à necessidade de informação dos glaucomatosos e à sua dificuldade de compreensão, decorrente do baixo nível cultural, de escolaridade e de fatores psicossociais relacionados à doença e condições de vida.
\end{abstract}

Glaucoma. Educação em saúde.

* Realizado no Núcleo de Prevenção de Cegueira da UNICAMP, em 1996.

Correspondência para/Correspondence to: Vital Paulino Costa - Av. Pacaembu, 1782 - 01234-000 São Paulo, SP - Brasil.

E-mail: vpcosta@ pop-gw.originet.com.br

Edição subvencionada pela FAPESP (Processo nº 97/09815-2).

Recebido em 13.1.1997. Reapresentado em 7.9.1997. Aprovado em 9.10.1997. 


\section{Abstract}

Purpose

To analyze the results of an educational program applied to glaucoma patients; to serve as a guideline for a definitive educational program to be developed at the Glaucoma Service of the University of Campinas.

Methods

Fifty glaucoma patients were given information about the disease and its treatment by an experienced nurse and answered the same questionnaire before and 2.7 months after the initial explanation. The technique of eyedrop instillation was compared before and after the educational program.

Results

There was no significant improvement concerning the knowledge of the identification of the ocular disease, the side effects, the meaning of glaucoma, the importance of treatment, the purpose of medications and the normal value of intraocular pressure. There was a significant improvement in all steps of eyedrop instillation $(p<0.01)$ and in knowledge regarding the importance of heredity $(p<0.001)$ and the purpose of perimetry $(p=0.033)$.

Conclusion Although a significant improvement was observed in the technique of eyedrop instillation, the authors conclude that this plan was not complete enough for its contents fully to be understood. These findings may be secondary to the vertical teaching-learning process, which is inappropriate for the patient, and to the difficulty in comprehending the information, which is associated with low cultural and instruction levels, and to psycosocial factors related to their disease.

Glaucoma. Health education.

\section{INTRO DU ÇÃO}

O glaucoma é uma afecção que, por suas características clínicas e prognóstico visual, requer a aderência do paciente ao tratamento e a realização de ações de auto-cuidado (atitudes favoráveis à sua própria saúde). Gerali e Diverde ${ }^{4}$ consideram que os profissionais da área de oftalmologia possuem papel importante na detecção precoce do glaucoma e na sua desmistificação. $\mathrm{O}$ desconhecimento sobre a doença e a elaboração de idéias falsas tendem a manter a pouca participação do paciente no tratamento, agravando o seu prognóstico visual.

Buscando analisar o conhecimento dos glaucomatosos sobre a sua doença e o seu tratamento, Costa e col. ${ }^{2}$ constataram que a população investigada estava desinformada sobre a suas condições clínicas, sobre a doença adquirida, sobre o seu tratamento e sobre os métodos para o diagnóstico e monitorização do glaucoma. Em outro estudo, Gullo e col. ${ }^{5}$ observaram altos índices de cegueira unilateral $(51,8 \%)$ e bilateral $(33,3 \%)$ em glaucomatosos atendidos pela primeira vez no Setor de Glaucoma da Universidade Estadual de Campinas. Os achados dessas investigações e a falta de orientação dos portadores de glaucoma apontaram para a necessidade de se desenvolver um programa de educação à saúde aos portadores de glaucoma.

O presente estudo foi realizado com os seguintes objetivos: 1- avaliar o resultado da implementação de um programa de educação à saúde a glaucomatosos; 2- fornecer subsídios para a implantação de um programa de educação à saúde aos portadores de glaucoma atendidos em um serviço universitário.

\section{MÉTO DO}

Realizado no Setor de Glaucoma de um hospital universitário de São Paulo, no período de março de 1994 a junho de 1995, participaram do trabalho 50 glaucomatosos de ambos os sexos, com idade igual ou superior a 16 anos e que atendiam aos seguintes critérios: ser portador de glaucoma e estar em tratamento ambulatorial. Previamente à coleta de dados, os portadores de glaucoma foram consultados sobre a disponibilidade $\mathrm{e}$ o consentimento para participar do estudo, sendo-lhes assegurado o anonimato dos registros.

Para a coleta de dados foi utilizado um formulário*, modificado a partir do instrumento de Costa e col. ${ }^{2}$,

* Os interessados poderão solicitar cópia do formulário, aos autores do presente trabalho. 
composto de uma parte inicial contendo informações relativas à identificação, além de questões destinadas à verificação do conhecimento sobre a sua doença e tratamento.

O trabalho foi realizado em duas etapas. Na primeira, foi feita uma entrevista individual com o paciente (utilizando-se o referido formulário), seguida de uma reunião na qual se aplicou o programa de educação à saúde por um dos autores (FAC), utilizando como recurso didático um olho anatômico. O conteúdo do programa estava embasado nos seguintes objetivos: 1- conscientizar o glaucomatoso para o significado da doença e importância do tratamento; 2- levantar as dificuldades e preocupações em relação à terapêutica; 3- orientar sobre as possíveis alternativas para a resolução de problemas; 4- orientar sobre o uso correto dos colírios e outros medicamentos e; 5- incentivar o autocuidado. Em relação ao uso dos colírios, procedia-se à demonstração da técnica utilizando-se um frasco de soro fisiológico $0,9 \%$ e, em seguida, solicitavase aos portadores de glaucoma que efetuassem a autoinstilação, orientando-os durante o procedimento. Os grupos eram constituídos de, no máximo, cinco glaucomatosos e seus acompanhantes. Cada paciente participava apenas de uma reunião, com duração média de uma hora.

Na segunda etapa, após o intervalo médio de 2,71 \pm 2,22 meses, realizou-se nova entrevista, onde os glaucomatosos foram submetidos ao mesmo questionário, por outra autora (JAGT) com o acréscimo de questões sobre a importância atribuída às orientações recebidas e sobre as modificações no autocuidado.

As entrevistas foram efetuadas em local reservado, procurando-se manter um ambiente adequado para a participação do glaucomatoso. A primeira etapa ocorreu, inicialmente, após a consulta oftalmológica. Entretanto, no decorrer do estudo, foi necessário realizá-la durante curva tensional diária, nos intervalos da medida da pressão intra-ocular (PIO).

As respostas dos portadores de glaucoma foram classificadas em "satisfatórias" ou "insatisfatórias", de acordo com critérios estabelecidos*. As respostas obtidas antes e após o programa de orientação foram comparadas através do teste de qui-quadrado ou do teste de Fisher. Valores de $\mathbf{p}$ inferiores a 0,05 foram considerados significantes.

\section{RESU LTAD O $S$}

$\mathrm{Na}$ Tabela 1 encontram-se os dados gerais da população estudada. Nela observa-se que a idade média dos glaucomatosos foi de 59,6 anos e que o grau de instrução era baixo $(74 \%$ dos portadores de glaucoma analfabetos ou com primeiro grau incompleto).

Na Tabela 2 são comparados os dados relativos ao conhecimento dos entrevistados sobre o glaucoma e seu tratamento, antes e após o programa de orientação. Essa Tabela revela que houve melhora significativa do conhecimento quanto à importância da hereditariedade e à finalidade da campimetria. Em relação ao conhecimento sobre o glaucoma, $68 \%$ declararam não ter recebido informações sobre a doença e $80 \%$ negaram terem sido informados sobre os efeitos colaterais das medicações. De uma maneira geral, a percentagem de respostas corretas após o programa de orientação aumentou significativamente de $47,2 \%$ para $61 \%(\mathrm{p}<0.0001)$. A percentagem de glaucomatosos que referia usar corretamente as medicações aumentou de $54 \%$ para $70 \%$, porém esta diferença não foi estatisticamente significante $(\mathrm{p}=0,180)$. Não houve melhora significativa no nível de respostas corretas em relação a todas as outras questões.

A Tabela 3 contém os dados relativos à técnica de instilação dos colírios, efetuada pelos portadores de glaucoma, antes e após o programa de orientação de enfermagem. Não foram incluídos nessa tabela sete glaucomatosos cujos familiares eram os responsáveis pela administração dos colírios. Dos 50 glaucomatosos, 45 (90\%) revelaram que não haviam sido previamente instruídos sobre o uso correto dos colírios.

Tabela 1 - Dados gerais da população de glaucomatosos estudada.

Table 1 - Demographics.

\begin{tabular}{|c|c|}
\hline Variável & $\begin{array}{l}\text { Número / Percentagem } \\
\qquad(n=50)\end{array}$ \\
\hline Idade média (anos) & $59,62 \pm 13,85$ \\
\hline $\begin{array}{l}\text { Tempo de } \\
\text { diagnóstico (anos) }\end{array}$ & $4,61 \pm 4,35$ \\
\hline $\begin{array}{l}\text { Nível de } \\
\text { escolaridade }\end{array}$ & $\begin{array}{l}\text { Analfabeto } 11(22 \%) \\
10 \text { Grau incompleto - } 26(52 \%) \\
10 \text { Grau completo - } 10(20 \%) \\
\text { 2 G rau incompleto - } 1(2 \%) \\
\text { 2 Grau completo - } 1(2 \%) \\
\text { Universitário - } 1(2 \%)\end{array}$ \\
\hline O cupação & $\begin{array}{l}\text { Aposentado - } 21(42 \%) \\
\text { Doméstica - } 17(34 \%) \\
\text { Outras - } 12(24 \%)\end{array}$ \\
\hline Sexo & $\begin{array}{l}\text { Feminino - } 26(52 \%) \\
\text { Masculino - } 24(48 \%)\end{array}$ \\
\hline Cor & $\begin{array}{l}\text { Branca - } 38(76 \%) \\
\text { Negra - } 9(18 \%) \\
\text { Parda - } 3(6 \%)\end{array}$ \\
\hline
\end{tabular}

* Os critérios poderão ser fornecidos a pedido dos interessados. 
Tabela 2 - Conhecimento dos entrevistados sobre glaucoma antes e após o programa de orientação.

Table 2- Knowledge about glaucoma before and after the educational program.

\begin{tabular}{|c|c|c|c|c|c|}
\hline \multirow{3}{*}{$\begin{array}{l}\text { Informação sobre o } \\
\text { conhecimento do entrevistado } \\
\text { Identificação da doença } \\
\text { ocular presente }\end{array}$} & \multicolumn{2}{|c|}{$\begin{array}{c}\mathrm{N} \text { ㅇ de respostas } \\
\text { antes da orientação }\end{array}$} & \multicolumn{2}{|c|}{$\begin{array}{l}\mathrm{N} 0 \text { de respostas } \\
\text { após a orientação }\end{array}$} & \multirow[t]{2}{*}{$P$} \\
\hline & Satisfatórias & $44(88 \%)$ & Satisfatórias & $43(86 \%)$ & \\
\hline & Insatistatórias & $06(12 \%)$ & Insatisfatórias & $07(14 \%)$ & 1,000 \\
\hline \multirow[t]{2}{*}{ O que é glaucoma } & Satisfatórias & $29(58 \%)$ & Satisfatórias & $35(70 \%)$ & \\
\hline & Insatisfatórias & $21(42 \%)$ & Insatisfatórias & $15(30 \%)$ & 0,297 \\
\hline \multirow[t]{3}{*}{ U so da medicação } & Regular & $27(54 \%)$ & Regular & $35(70 \%)$ & \\
\hline & Irregular & $14(28 \%)$ & Irregular & $07(14 \%)$ & \\
\hline & Sem prescrição & $09(18 \%)$ & Sem prescrição & $08(16 \%)$ & 0,180 \\
\hline \multirow{2}{*}{$\begin{array}{l}\text { Efeitos colaterais } \\
\text { dos medicamentos }\end{array}$} & Satisfatórias & $13(26 \%)$ & Satisfatórias & $15(30 \%)$ & \\
\hline & Insatisfatórias & $37(74 \%)$ & Insatisfatórias & $35(70 \%)$ & 0,823 \\
\hline \multirow[t]{2}{*}{ Importância do tratamento } & Satisfatórias & $38(76 \%)$ & Satisfatórias & $43(86 \%)$ & \\
\hline & Insatisfatórias & $12(24 \%)$ & Insatisfatórias & $07(14 \%)$ & 0,307 \\
\hline \multirow{2}{*}{$\begin{array}{l}\text { Finalidade } \\
\text { dos medicamentos }\end{array}$} & Satisfatórias & $36(72 \%)$ & Satisfatórias & $30(60 \%)$ & \\
\hline & Insatisfatórias & $14(28 \%)$ & Insatisfatórias & $20(40 \%)$ & 0,291 \\
\hline \multirow[t]{2}{*}{ Valor da última PIO } & Sabia & $15(30 \%)$ & Sabia & $24(48 \%)$ & \\
\hline & Não sabia & $35(70 \%)$ & Não sabia & $26(52 \%)$ & 0,100 \\
\hline \multirow[t]{2}{*}{ Valor normal da PIO } & Satisfatórias & $15(30 \%)$ & Satisfatórias & $21(42 \%)$ & \\
\hline & Insatisfatórias & $35(70 \%)$ & Insatisfatórias & $29(58 \%)$ & 0,297 \\
\hline \multirow{2}{*}{$\begin{array}{l}\text { Importância da } \\
\text { hereditariedade }\end{array}$} & Sabia & $10(20 \%)$ & Sabia & $37(74 \%)$ & \\
\hline & Não sabia & $40(80 \%)$ & $\mathrm{N}$ ão sabia & $13(26 \%)$ & $<0,001^{*}$ \\
\hline \multirow{2}{*}{$\begin{array}{l}\text { Finalidade da } \\
\text { campimetria }\end{array}$} & Satisfatórias & $11(22 \%)$ & Satisfatórias & $22(44 \%)$ & \\
\hline & Insatisfatórias & $39(78 \%)$ & Insatisfatórias & $28(56 \%)$ & $0,033 *$ \\
\hline
\end{tabular}

Observa-se na Tabela 3 que houve melhora significativa em todos os passos da técnica de instilação de colírios $(\mathrm{p}<0,05)$. De uma maneira geral, houve aumento significativo nos passos corretos de instilação de $23,2 \%$ para $69,2 \%(p<0,0001)$. A maioria dos portadores de glaucoma $(n=45)$ considerou relevantes as informações recebidas através do programa de orientação de enfermagem, equanto 34 afirmaram que começaram a tratar-se de forma diferente.

\section{DISCUSSÃO}

Sabe-se que aproximadamente metade dos casos de cegueira é prevenível ou reversível, tanto nos EUA $^{12}$ como no Brasil ${ }^{8}$. Chiang e col. ${ }^{1}$ demonstraram que um ano de cegueira em um indivíduo adulto produtivo custava ao governo norte-americano cerca de U\$12.000,00, em 1990. Além disso, o custo de preven-ção da cegueira é muito menor que o custo causado pela cegueira ${ }^{6}$. No presente estudo,

Tabela 3 - Técnica de instilação de colírios em portadores de glaucoma antes e após o programa de orientação $(n=43)$

Table 3 - Technique of eyedrop instillation before and after the educational program $(n=43)$.

\begin{tabular}{llrlll}
\hline Procedimento relativo a & \multicolumn{2}{c}{ Antes da orientação } & \multicolumn{2}{c}{ Após a orientação } & $P$ \\
\hline Número de gotas & 1 gota & $21(48,8 \%)$ & 1 gota & $34(79,1 \%)$ & \\
instilado & 2 ou mais gotas & $22(51,2 \%)$ & 2 ou mais gotas & $09(20,9 \%)$ & $0,007^{*}$ \\
Local de instilação & Correto & $15(34,9 \%)$ & Correto & $36(83,7 \%)$ & \\
& Incorreto & $28(65,1 \%)$ & Incorreto & $07(16,3 \%)$ & $<0,001^{*}$ \\
Movimento de piscar & Correto & $08(18,6 \%)$ & Correto & $33(76,7 \%)$ & \\
após a instilação & Incorreto & $35(81,4 \%)$ & Incorreto & $10(23,3 \%)$ & $<0,001^{*}$ \\
Ato de fechar o olho & Correto & $04(9,3 \%)$ & Correto & $27(62,8 \%)$ & \\
após a instilação & Incorreto & $39(91,7 \%)$ & Incorreto & $16(37,2 \%)$ & $<0,001^{*}$ \\
O clusão do & Correto & - & Correto & $22(51,2 \%)$ & \\
ponto lacrimal & Incorreto & $43(100 \%)$ & Incorreto & $21(48,8 \%)$ & $<0,001^{*}$ \\
\hline
\end{tabular}

$*-p<0,05$ 
investigou-se a eficácia de um programa educacional em aumentar o conhecimento de glaucomatosos sobre sua doença, o que poderia resultar em melhora da aderência ao tratamento e retardo da progressão da doença.

A participação do paciente em apenas uma reunião, na qual foi aplicado o programa de orientação, revelou ser insuficiente para a aquisição de grande parcela do conteúdo exposto, principalmente o relacionado aos aspectos cognitivos. Esses resultados podem ser conseqüentes ao tipo de processo ensino-aprendizagem, desenvolvido de forma vertical, que não atende à necessidade de informação dos glaucomatosos e à sua dificuldade de compreensão, decorrente do baixo nível cultural, de escolaridade e fatores psicossociais relacionados à doença e condições de vida.

Observa-se, também, que não houve mudança significativa na regularidade do uso dos medicamentos. O tratamento prolongado, sem a compreensão da sua finalidade e importância, dificultam a participação no tratamento. Os glaucomatosos, ao serem questionados sobre a freqüência com que usavam os medicamentos, tendiam a repetir os intervalos horários indicados pelo médico. Entretanto, ao serem interrogados sobre os horários, percebia-se que esses intervalos não eram respeitados. Apesar disso, muitos confiavam no tratamento e tinham a falsa expectativa de que, através dos colírios, obteriam melhora da acuidade visual, o que também contribui para reduzir o envolvimento com o tratamento.

Outros fatores podem estar associados à irregularidade do tratamento. Dentre eles destacamse: a falta de condições econômicas ${ }^{2}$, o esquecimento dos horários dos medicamentos, a não melhora da visão, os efeitos colaterais e a incapacidade de realizar a auto-instilação dos colírios ${ }^{3}$. A aquisição de conhecimento sobre a importância da hereditariedade parece ter chamado a atenção dos portadores de glaucoma, provavelmente pelo fato deste conhecimento explicar pelo menos em parte o seu problema e, além disto, alertar para o risco de seus descendentes. A mudança verificada na técnica de instilação do colírio pode ser consequiência da participação mais ativa do glaucomatoso no seu tratamento, proporcionada por uma troca de conhecimentos e sentimentos em relação à doença favorecida por este momento de reunião. $\mathrm{O}$ mesmo pode ser observado em relação à campimetria, o que comprova a melhor compreensão dos aspectos práticos do programa educativo.

O programa de orientação possibilitou a absorção de apenas parte do seu conteúdo, especialmente àquela relacionada à técnica de administração dos colírios. Ley ${ }^{10}$ observou que os pacientes tendem a esquecer de um terço à metade das informações recebidas, minutos após sua aquisição. Rosenthal e col. ${ }^{11}$ investigaram o efeito de um vídeo de $6 \mathrm{~min}$. sobre o conhecimento a respeito do glaucoma. Apesar de uma melhora ter sido observada imediatamente após a apresentação do vídeo, após seis meses houve uma queda importante do nível de conhecimento. Kim e cols. ${ }^{9}$ demonstraram que um vídeo de 12 minutos produzido pela Academia Americana de Oftalmologia promoveu uma melhora do conhecimento sobre glaucoma após uma semana, mas não após três meses. Assim como o presente estudo, os autores citados acima constataram que uma única exposição do indivíduo à orientação sobre o glaucoma é ineficaz do ponto de vista educativo.

Partindo do pressuposto que o conhecimento não corresponde a um acúmulo gradual de informações transmitidas, mas, sim, a um processo construído na interação entre o sujeito e o outro, os autores sugerem que a orientação dos portadores de glaucoma possa ser feita de forma contínua e progressiva, levandose em conta as concepções e significados que atribuem à sua doença e tratamento. A utilização de folhetos ou de vídeos contendo informações básicas sobre a doença e seu tratamento constituem estratégias adicionais que podem servir para conscientizar o glaucomatoso sobre a importância do tratamento do glaucoma. Um programa de educação à saúde dos glaucomatosos com essas características é fundamental para a prevenção da cegueira e deverá ser aplicado não só em nível de hospitais universitários, gerais e centros de saúde, mas também de rotina nos consultórios oftalmológicos.

É sempre importante enfatizar que o modelo biomédico, com ênfase na doença e não na saúde, representa apenas parte das formas possíveis de intervenção social na saúde pública, que incluem também a abordagem educativa, de mudança de comportamento, a abordagem centralizada no cliente e a abordagem societária. Programas de educação em saúde, como esse desenvolvido pelo hospital universitário, visam a desencadear mudanças de comportamento individual e poderão servir de base para a instalação de promoção em saúde, cujo intuito principal é o de promover mudanças de comportamento organizacional ${ }^{13}$.

Esta linha de pesquisa de saúde pública, que vem sendo estimulada pelo Núcleo de Prevenção de Cegueira da UNICAMP ${ }^{7}$, deve ser colocada como uma das prioridades em saúde ocular neste mo- 
mento. Após ter sido constatado que o nível de conhecimento dos portadores de glaucoma sobre a doença é insuficiente ${ }^{2}$, que a técnica de instilação dos colírios utilizados no tratamento do glaucoma é inadequada ${ }^{3}$, e que os portadores de glaucoma chegam ao Serviço Universitário tardiamente ${ }^{5}$, verificou-se que o programa de educação proposto mostrou-se insuficiente para a apreensão de todo o seu conteúdo cognitivo. Tradicionalmente, tem-se enfatizado pouco a orientação de portadores

\section{REFERÊNCIAS}

1. CHIANG, Y.P. et al. Federal budgetary cost of blindness. Milbank Q., 70:319-40, 1992.

2. COSTA, V.P. et al. O que os pacientes sabem sobre glaucoma? Arq. Bras. Oftalmol., 58: 36-41, 1995.

3. COSTA, V.P. et al. Análise da técnica de instilação de colírios em pacientes glaucomatosos. Rev. Bras. Oftalmol., 54:523-8, 1995.

4. GERALI, P.S. \& DIVERDE, M. Preventing blindness. $J$. Ophthal. Nurs. Technol., 10:34, 1991.

5. GULLO, R.M. et al. Condições oculares de pacientes glaucomatosos em um Hospital Universitário. Arq. Bras. Oftalmol., 59:147-50, 1996.

6. JAVITT, J.C. et al. Detecting and treating retinopathy in patients with type I diabetes mellitus: savings associated with improved implementation of current guidelines. Ophthalmology, 98:156574, 1991.

7. KARA-JOSÉ, N. et al. Criação do Núcleo de Prevenção de de doenças oculares crônicas em instituições públicas. Procurou-se aplicar modelo educativo utilizando tecnologia simples e reprodutível ao alcance de serviços que se disponham a implementar e desenvolver após-consulta. Um novo programa de educação à saúde, realizado de forma contínua e progressiva, através de várias reuniões e fornecimento de material educativo aos glaucomatosos e seus parentes, deverá ser desenvolvido e avaliado.

Cegueira. Arq. Bras. Oftalmol., 50:145-7, 1987.

8. KARA-JOSÉ, N. et al. Screening and surgical results from Cataract Free-Zone Project in Campinas, Brazil, and Chimbot, Peru. Int. Ophthalmol., 14:155-64, 1990.

9. KIM, S. et al. Glaucoma patient education. Invest. Ophthalmol. Vis. Sci., 37(Suppl.):S642, 1996.

10. LEY, P. Psychology of compliance. Br. J. Soc. Clin. Psychol., 18: 245-8, 1979.

11. ROSENTHAL, A.R. et al. Educating the glaucoma patient. $\mathrm{Br}$. J. Ophthalmol., 67:814-7, 1983.

12. SOMMER, A. et al. Racial differences in the cause-specific prevalence of blindness in East Baltimore. N. Engl. J. Med., 325:1412-7, 1991.

13. CANDEIAS, N. M. F. Conceitos de educação e de promoção em saúde: mudanças individuais e mudanças organizacionais. Rev. Saúde Pública, 31:209-13, 1997. 